\title{
Serum prolidase activity is associated with non-diabetic metabolic syndrome
}

\author{
Suzan Tabur ${ }^{1}$, Elif Oguz ${ }^{2}$, Mehmet Ali Eren ${ }^{3}$, Hakan Korkmaz ${ }^{*}$, Esen Savas ${ }^{4}$, Nurten Aksoy ${ }^{5}$ and Tevfik Sabuncu ${ }^{3}$
}

\begin{abstract}
Objective: The aim of this study was to determine the role of serum prolidase activity and the possible association with oxidative stress parameters in non-diabetic metabolic syndrome.

Methods: 30 obese patients without metabolic syndrome (MetS), 34 non-diabetic obese patients with MetS, and 23 volunteer control subjects were enrolled in the study. Fasting plasma glucose (FPG), plasma glucose following $75 \mathrm{~g}$ glucose administration, high-density lipoprotein- cholesterol (HDL-C), high-density lipoprotein- cholesterol (LDL-C), total cholesterol, triglyceride (TG), total antioxidant status (TAS), total oxidative status (TOS), oxidative stress index (OSI), and prolidase activities of all subjects were analyzed.
\end{abstract}

Results: Prolidase levels was significantly higher in MetS group compared to both obese and control groups $(p<0.001$ and $p<0.05$ respectively). Prolidase was also higher in the obese group than in the control group $(p<0.05)$. Prolidase was negatively correlated with TAS and HDL-C $(r=-0,362, p<0.001 ; r=-0.320, p<0.01$, respectively) and positively correlated with BMI, weight, waist-c, SBP, DBP, TG, TC, LDL-C.

Conclusion: Prolidase activity may have a role in the pathogenesis of metabolic syndrome.

Keywords: Metabolic syndrome, Non-diabetic, Obesity, Prolidase

\section{Introduction}

Metabolic syndrome (MetS) is defined as the existence of obesity, insulin resistance, glucose intolerance, hypertension, and dyslipidemia [1]. Subjects with MetS may be obese but all obese patients may not have MetS. Both MetS and obesity have been shown to have impacts on cardiovascular mortality and morbidity [2].

Endothelial disfunction causes alterations in the arterial vasculature and leads to micro- and macrovascular complications. The remodelling of the endothelial basal membrane, resulted with erosion and thrombosis, increases the oxidative stress and alters matrix metalloproteinases (MMPs) expression [3].

Prolidase, a member of the MMP family, is a cytosolic imidodipeptidase, which specifically splits imidodipeptides with C-terminal proline or hydroxyproline. The enzyme plays an important role in the recycling of proline from imidodipeptides for resynthesis of collagen and other proline containing proteins [4]. Prolidase enzyme

\footnotetext{
* Correspondence: drhkorkmaz@yahoo.com.tr

${ }^{1}$ Faculty of Medicine, Department of Internal Medicine, Division of Endocrinology, Gaziantep University, 27100, Sahinbey, Gaziantep, Turkey Full list of author information is available at the end of the article
}

activity has been shown in plasma, erythrocytes, leukocytes, dermal fibroblasts and various organs such as kidney, brain, heart, thymus, uterus, lung, spleen and pancreas $[5,6]$. It is demonstrated that the activity of this enzyme may have a role in various disorders such as chronic liver disease, osteoporosis, osteoarthritis, uraemia, and hypertension [7-11]. To the best of our knowledge, there is no data concerning the serum prolidase activity in metabolic syndrome. Therefore, the aim of this study was to determine the role of serum prolidase activity in non-diabetic metabolic syndrome.

\begin{abstract}
Method
Subjects

Patients who were admitted for the evaluation of obesity were recruited from the Endocrinology and Internal Medicine outpatient clinic. A standard $75 \mathrm{~g}$ oral glucose tolerance test (OGTT) was administered to all participants, and patients were randomized to three groups according to their affected glucose metabolism. Groups included 30 obese patients without MetS and glucose intolerance (mean age $33.67 \pm 7.9$ years, $2 \mathrm{M}$ and $28 \mathrm{~F}$ ), 34
\end{abstract}


non-diabetic obese patients with MetS (mean age 35.18 \pm 6.8 years, $3 \mathrm{M}$ and $31 \mathrm{~F}$ ), and 23 sex and age- matched healthy control subjects (mean age32.39 \pm 4.7 years, $3 \mathrm{M}$ and 20F).

Although the MetS group was composed of nondiabetics, all the patients had varying degrees of glucose intolerance or were insulin resistant. The control group had normal OGTT. MetS is defined according to the criteria accepted in the Third Report of the National Cholesterol Education Program (NCEP) [12]. Hypertension and hyperlipidemia were diagnosed for the first time at the initiation of the study, so no participant was using an antihypertensive or anti-lipidemic drug before obtaining the blood samples. Subjects having diabetes, heart failure, cirrhosis, infection, renal failure, pregnancy or malignancy; those on antioxidants such as antihypertensive medications, lipid-lowering medications, and vitamin E; and smokers were excluded.

Age, weight, height, body mass index (BMI: body weight $(\mathrm{kg}) /$ height $\left.(\mathrm{cm})^{2}\right)$, and systolic (SBP) and diastolic blood pressures (DBP) of all subjects were recorded. Fasting plasma glucose (FPG), plasma glucose following $75 \mathrm{~g}$ glucose administration, high density lipoprotein- cholesterol (HDL-C), Low density lipoprotein-cholesterol (LDL-C), total cholesterol (TC), triglyceride (TG), total antioxidant status (TAS), total oxidative status (TOS), oxidative stress index (OSI), and prolidase activities of all subjects were analyzed. The study was approved by the local ethics committee, and all participants gave signed informed consent.

\section{Blood samples and preparation}

Blood samples were drawn after overnight fasting, and serum samples were stored at $-80^{\circ} \mathrm{C}$ until biochemical determination of TAS, TOS and prolidase activities.

\section{Measurement of total antioxidant status}

Serum TAS was determined using a novel automated measurement method developed by Erel [12]. In the method, hydroxyl radical, the most potent biological radical, is produced first. In the assay, reagent 1 containing ferrous ion solution is mixed with reagent 2 , which contains hydrogen peroxide. The sequentially produced radicals, such as brown colored dianisidinyl radical cation produced by the hydroxyl radical, are also potent radicals. The anti-oxidative effect of the study sample against the potent-free radical reactions, which are initiated by the produced hydroxyl radical, is measured. The assay has excellent precision values, lower than $3 \%$, and the results are expressed as mmol Trolox Equiv./l.

\section{Measurement of total oxidant status}

Serum TOS was determined using a novel automated measurement method developed by Erel [13]. Oxidants present in the study sample oxidize the ferrous ion-o-dianisidine complex to ferric ion. The oxidation is enhanced by glycerol molecules, which are abundantly present in the reaction medium. The ferric ion makes a colored complex with xylenol orange in an acidic medium. The color intensity, which can be measured spectrophotometrically, is related to the total amount of oxidant molecules present in the sample. The assay is calibrated with hydrogen peroxide, and the results are expressed as $\mu \mathrm{mol} \mathrm{H}_{2} \mathrm{O}_{2}$ Equiv./l.

\section{Oxidative stress index}

Percent ratio of TOS to TAS level was accepted as OSI (OSI (Arbitrary Unit) $=$ TOS $\left(\mu \mathrm{mol} \mathrm{H}_{2} \mathrm{O}_{2}\right.$ Equiv./l)/TAS (mmol Trolox Equiv./l)) [10].

\section{Prolidase measurement}

Prolidase activity was determined by a photometric method based on the measurement of the proline levels produced by prolidase [14].

Serum samples $(100 \mu \mathrm{l})$ were mixed with $100 \mu \mathrm{l}$ of serum physiological. A total of $25 \mu \mathrm{l}$ of the mixture was preincubated with $75 \mathrm{ml}$ of the preincubation solution (50 $\mathrm{mmol} / \mathrm{l}$ Tris $\mathrm{HCl}$ buffer $\mathrm{pH} 7.0$ containing $1 \mathrm{mmol} / \mathrm{l}$ glutathione, $50 \mathrm{mmol} / \mathrm{l} \mathrm{MnCl}_{2}$ ) at $37^{\circ} \mathrm{C}$ for $30 \mathrm{~min}$. The reaction mixture, which contained $144 \mathrm{mmol} / \mathrm{l}$ gly-pro, $\mathrm{pH} 7.8$ $(100 \mathrm{ml})$, was incubated with $100 \mathrm{ml}$ of preincubated sample at $37^{\circ} \mathrm{C}$ for $5 \mathrm{~min}$. To stop the incubation reaction, 1 $\mathrm{ml}$ glacial acetic acid was added. After adding $300 \mathrm{ml}$ Tris $\mathrm{HCl}$ buffer, $\mathrm{pH} 7.8$, and $1 \mathrm{ml}$ ninhydrin solution $(3 \mathrm{~g} / \mathrm{dl}$ ninhydrin was melted in $0.5 \mathrm{~mol} / \mathrm{l}$ orthophosphoric acid), the mixture was incubated at $90^{\circ} \mathrm{C}$ for $20 \mathrm{~min}$ and cooled with ice. Absorbance was then measured at a $515 \mathrm{~nm}$ wavelength to determine proline value.

Intraassay and interassay coefficient of variations (CVs) were lower than $10 \%$. We measured the serum prolidase activity by the method optimized by Gültepe [15], which is a modification of Myara and Chinard's methods [16,17] based on the spectrophotometric determination of proline levels liberated from glycyl-L-proline by prolidase enzyme.

Plasma TG, total cholesterol, LDL-C, and HDL-C concentrations were measured by automated chemistry analyzer (Aeroset, Abbott) using commercial kits (Abbott).

\section{Statistical analysis}

Continuous variables were expressed as mean \pm S.D, and non-parametric data were expressed as median and ranges. Categorical data were compared by Chi-square tests. Oneway ANOVA was used for multiple comparisons among the groups, and the LSD test was used if any statistical significance was found. Normality of distribution was evaluated with the Kolmogorov-Smirnov test. Pearson correlation test was used to evaluate any relationships between parameters. All statistical tests were two-sided. $\mathrm{P}<0.05$ was regarded as significant for all analysis. All analyses were conducted using SPSS 11.5 (SPSS for Windows 11.5, Chicago, IL, USA). 


\section{Results}

Mean ages of the three groups were similar. SBP, DBP and TG levels were significantly higher in the MetS group compared to both obese and control groups (all $\mathrm{p}<0.001$ ) (Table 1). Both obese and MetS groups had significantly higher BMI levels than the control group (all $\mathrm{p}<0.001$ and $\mathrm{p}<0.001$ respectively). The obese group had higher SBP and DBP than the control group (all $\mathrm{p}<0.001$ ). MetS group had significantly lower HDL-C levels than the control group $(\mathrm{p}<0.001)$. HDL-C levels was also lower in the obese group than in the control group, but the difference was not significant. In the MetS group 10 patients had only impaired fasting glucose (IFG), 2 impaired glucose tolerance (IGT), 9 had both IFG and IGT and 13 were only insülin resistant. Prolidase levels were significantly higher in MetS group compared to both obese and control groups $(\mathrm{p}<$ 0.001 and $\mathrm{p}<0.05$ respectively) and also in the obese group compared to the control group $(\mathrm{p}<0.05)$. TAS was lower in both MetS and obese groups than in the control group ( $\mathrm{p}<0.001$ and $\mathrm{p}<0.05$ respectively). There wasn't any significant difference according to BMI levels between MetS and obese groups. OSI was significantly higher in both obese and MetS groups than in the control group $(\mathrm{p}<$ 0.001 and $p<0.001$ respectively). The clinical and biochemical data are shown in Table 1.

In correlation analysis, prolidase was negatively correlated with TAS and HDL-C $(r=-0,362, p<0.001 ; r=-0.320$, $\mathrm{p}<0.01$ ) and positively correlated with BMI, weight, waist-c, SBP, DBP, TG, TC, LDL-C $(r=0.330, p=0.002 ; r=0.298$ $\mathrm{p}=0.006 ; \mathrm{r}=0.4 \mathrm{p}<0.001 ; \mathrm{r}=0.367, \mathrm{p}=0.001 ; \mathrm{r}=0.358$, $\mathrm{p}=0.001 ; \mathrm{r}=0.293, \mathrm{p}=0.008 ; \mathrm{r}=0.297, \mathrm{p}=0.006 ; \mathrm{r}=0.306$, $\mathrm{p}=0.005$, respectively; Table 2 ). These associations were confirmed in the multiple regression analysis $\left(R^{2}=0,226\right.$, $\mathrm{p}=0,001$ ). In multivariate logistic regression analysis prolidase activity was found to be an important predictor for MetS (A one unit change in prolidase would make the MetS 1,115 as likely to ocur; $\left.R^{2}=0,324, p=0,001\right)$.

\section{Discussion}

In this study, we investigated the possible association between serum prolidase activity and non-diabetic metabolic syndrome.We found a significant increase in serum prolidase activity, a member of MMPs, in patients with non-diabetic metabolic syndrome compared to obese or healthy control groups. A significant correlation of serum prolidase activity was also determined both with increased BMI, weight, waist-c, SBP, DPB, TG, TC, LDL$\mathrm{C}$ and decreased TAS and HDL-C levels.

MetS is defined as the existance of obesity and at least other two factors among hypertension, dyslipidemia and diabetes mellitus or glycemia of $>100 \mathrm{mg} / \mathrm{dl}$ [3]. Endothelial dysfunction in MetS leads cardiovascular risk accompained by high morbidity and mortality. Increased oxidative stress and altered MMPs are shown two of the factors that play in the pathogenesis of MetS. Prolidase, a member of MMPs, plays an important role in collagen metabolism and extracellular matrix remodeling $[4,18]$. Prolidase enzyme activity has been investigated in various disorders such as chronic liver disease [7], osteoporosis [8], osteoarthritis [9], uremia [10], diabetic neuropathy [19], hypertension [11], coronary

Table 1 Clinical and metabolic parameters of MetS, Obese and control groups

\begin{tabular}{|c|c|c|c|}
\hline & MetS $(n=34,31 M$ and $3 F)$ & Obese $(n=30,28 M$ and $2 F)$ & Control $(n=23,20 M$ and $3 F)$ \\
\hline Age (years) & $35.18 \pm 6.8$ & $33.67 \pm 7.9$ & $32.39 \pm 4.7$ \\
\hline Body weight (kg) & $97.27 \pm 12.6^{b, f}$ & $92.07 \pm 19.2^{c}$ & $60.30 \pm 10.3$ \\
\hline $\mathrm{BMI}(\mathrm{kg} / \mathrm{m} 2)$ & $38.91 \pm 5.5^{b}$ & $36.59 \pm 5.2^{c}$ & $22.93 \pm 3.4$ \\
\hline Waist-C (cm) & $106.85 \pm 10.3^{b, f}$ & $99.73 \pm 13.2^{c}$ & $75.52 \pm 8.1$ \\
\hline $\mathrm{SBP}(\mathrm{mmHg})$ & $136.77 \pm 12.0^{a, b}$ & $118.67 \pm 13.1^{c}$ & $105.65 \pm 7.7$ \\
\hline $\mathrm{DBP}(\mathrm{mmHg})^{*}$ & $90(70-110)^{a, b}$ & $80(70-100)^{c}$ & $60(50-80)$ \\
\hline $\mathrm{TC}(\mathrm{mg} / \mathrm{dl})$ & $194.39 \pm 35.6^{e}$ & $181.66 \pm 24.2$ & $168.13 \pm 24.7$ \\
\hline $\mathrm{HDL}(\mathrm{mg} / \mathrm{dl})$ & $44.39 \pm 13.3 b$ & $42.14 \pm 9.9$ & $55.65 \pm 5.2$ \\
\hline TG (mg/dl) & $174.91 \pm 72.9^{a, b}$ & $100.07 \pm 35.3$ & $73.44 \pm 30.6$ \\
\hline $\mathrm{LDL}(\mathrm{mg} / \mathrm{dl})$ & $113.68 \pm 37.4$ & $117.76 \pm 23.4^{h}$ & $97.80 \pm 21.9$ \\
\hline TOS (mmol H2O2 Equiv./l) & $13.94 \pm 2.19^{e, f}$ & $12.74 \pm 2.1$ & $13.05 \pm 2.3$ \\
\hline TAS (mmol Trolox Equiv./l) & $0.95 \pm 0.1^{a, b}$ & $1.10 \pm 0.1^{\mathrm{h}}$ & $1.16 \pm 0.1$ \\
\hline OSI (arbitrary unit) & $14.67 \pm 2.2^{a, b}$ & $11.48 \pm 2.3$ & $10.65 \pm 2.4$ \\
\hline $\mathrm{FPG}(\mathrm{mg} / \mathrm{dl})$ & $102.88 \pm 13.3^{b}$ & $97.94 \pm 7.4^{\mathrm{h}}$ & $90.87 \pm 9.8$ \\
\hline Prolidase & $708.93 \pm 10.4^{b, f}$ & $703.17 \pm 8.1^{h}$ & $696.69 \pm 11.5$ \\
\hline
\end{tabular}

BMI, body mass index; DBP, diastolic blood pressure; FPG, fasting plasma glucose; HDL, high-density lipoprotein; LDL, low-density lipoprotein; MetS, metabolic syndrome; OSI, oxidative stress index SBP, systolic blood pressure; TAS, total antioxidant status; TC, total cholesterol;TG, triglyceride; TOS, total oxidative status; Waist- $C$, waist circumference. ${ }^{*}$ Data in which non-parametric tests were used and expressed as median (range). $P<0.001$ : ${ }^{a}$ Met $S$ versus obese; ${ }^{b}$ MetS versus control; ${ }^{c}$ obese versus control. $\mathrm{P}<0.01$ : ${ }^{\mathrm{d}}$ MetS versus obese; ${ }^{\mathrm{e}}$ MetS versus control. $\mathrm{P}<0.05$ : ${ }^{\mathrm{f}}$ MetS versus obese; ${ }^{\mathrm{g}}$ MetS versus control; ${ }^{\mathrm{h}}$ obese versus control. 
Table 2 Correlations between prolidase, and other clinical and metabolic parameters

\begin{tabular}{|c|c|c|c|c|c|c|c|c|c|c|}
\hline & BMI & WEIGHT & WAIST-C & SBP & DBP & TG & TC & HDL-C & LDL-C & TAS \\
\hline \multicolumn{11}{|c|}{ PROLIDASE } \\
\hline R & 0.330 & 0.298 & 0.400 & 0.367 & 0.358 & 0.293 & 0.297 & $-0,320$ & 0,306 & $-0,362$ \\
\hline P & 0.002 & 0.006 & $<0.001$ & ,001 & ,001 & ,008 & ,006 & ,003 & ,005 & ,001 \\
\hline \multicolumn{11}{|c|}{ BMI } \\
\hline$R$ & & 0.925 & 0.892 & 0.591 & 0.675 & 0.423 & 0.245 & -0.304 & 0.170 & -0.437 \\
\hline P & & $<0.001$ & $<0.001$ & $<0.001$ & $<0.001$ & $<0.001$ & 0.023 & 0.004 & 0.125 & $<0.001$ \\
\hline \multicolumn{11}{|c|}{ WEIGHT } \\
\hline$R$ & & & 0.915 & 0.581 & 0.669 & 0.427 & 0.311 & -0.302 & 0.234 & -0.406 \\
\hline P & & & $<0.001$ & $<0.001$ & $<0.001$ & $<0.001$ & 0.004 & 0.005 & 0.034 & $<0.001$ \\
\hline \multicolumn{11}{|c|}{ WAIST-C } \\
\hline$R$ & & & & 0.594 & 0.675 & 0.481 & 0.322 & -0.292 & 0.220 & -0.434 \\
\hline P & & & & $<0.001$ & $<0.001$ & $<0.001$ & 0.002 & 0.006 & 0.046 & $<0.001$ \\
\hline \multicolumn{11}{|c|}{ SBP } \\
\hline$R$ & & & & & 0.844 & 0.436 & 0.298 & -0.197 & 0.161 & -0.494 \\
\hline P & & & & & $<0.001$ & $<0.001$ & 0.005 & 0.069 & 0.147 & $<0.001$ \\
\hline \multicolumn{11}{|c|}{ DBP } \\
\hline$R$ & & & & & & 0.382 & 0.333 & -0.217 & 0.218 & -0.409 \\
\hline P & & & & & & $<0.001$ & 0.002 & 0.044 & 0.048 & $<0.001$ \\
\hline \multicolumn{11}{|c|}{ TG } \\
\hline$R$ & & & & & & & 0.351 & -0.166 & -0.033 & -0.379 \\
\hline P & & & & & & & 0.001 & 0.133 & 0.767 & $<0.001$ \\
\hline \multicolumn{11}{|c|}{ TK } \\
\hline$R$ & & & & & & & & -0.006 & 0.849 & -0.326 \\
\hline$P$ & & & & & & & & 0.954 & $<0.001$ & 0.002 \\
\hline \multicolumn{11}{|c|}{ HDL-C } \\
\hline$R$ & & & & & & & & & -0.338 & 0.123 \\
\hline$P$ & & & & & & & & & 0.002 & 0.264 \\
\hline \multicolumn{11}{|c|}{ LDL-C } \\
\hline$R$ & & & & & & & & & & -0.225 \\
\hline$P$ & & & & & & & & & & 0.042 \\
\hline
\end{tabular}

BMI, body mass index; DBP, diastolic blood pressure; HDL, high-density lipoprotein; LDL, low-density lipoprotein; MetS, metabolic syndrome; SBP, systolic blood pressure; TG, triglyceride; Waist-C, waist circumference; TAS, total antioxidant status.

artery disease [20], and ovarian cancer [21]. There are some studies revealing the role of MMPs in MetS. Goncalves et al. reported an increase in pro-MMP-9, MMP-8 and TIMP-1 levels while without any difference in MMP-2, MMP-3 and TIMP-2 levels compared to healthy controls [22]. Additionally, an increase in MMP-8 levels in MetS patients [23] and elevated levels of MMP-2 activity, but not of MMP-9 in non-diabetic MetS [24] was reported. On the other hand, there are some studies in the literature regarding MMPs profile in obesity [24-29], diabetes mellitus [30-33] hypertension [34-36] and dyslipidemia [37,38], clinical conditions representing diagnostic criteria for the definition of the metabolic syndrome.
We have shown previously that MetS and obesity may alter oxidative stress, which contributes to atherosclerosisrelated cardiovascular events [39]. In this study we also found a significant increase $(p<0.001)$ of OSİ levels and a significant decrease $(\mathrm{p}<0.001)$ of TAS levels in metabolic syndrome and non-diabetic Mets group compared to obese and healthy control groups similar to our previous study [39].

We eveluated firstly serum prolidase activity in nondiabetic metabolic syndrome and demonstrated its elevation in this patient group. Additionally, the correlation analysis showed that prolidase activity had a significant positive correlation with BMI, weight, waist-c, SBP, DBP, 
TG, TC, LDL-C and inversely negative correlation with TAS and HDL-C in our study. Correlation between serum prolidase activity and markers of oxidative stress paremeters in this study suggests the association of collagen turnover and oxidative stress in non-diabetic MetS.

Serum prolidase activity was significantly higher in MetS group compared to the only obese group. This may be resulted from that hypertension, hypertriglyceridemia, low HDL-C levels, IGT, IFG and insülin resistance, are found more frequently in MetS compared to obesity. Logistic regression analysis demonstrated that prolidase activity was an important predictor for MetS as for the last point of this study.

Demirbağ et al. [11] has found a significant correlation between prolidase activity and presence and duration of hypertension supporting our data. Yıldız et al. [20] also showed that serum prolidase activity was positively correlated with presence of hypertension, SBP and inversely correlated with HDL-C levels. Hilali et al. [18] reported that elevated serum prolidase activity and oxidative stress may be associated with increased cardiovascular risk in polycystic ovary syndrome and/or menstrual irregularities associated with this syndrome. Serum prolidase activity was suggested as a marker of osteoporosis in type 2 diabetes mellitus [8].

Consequently we suggest that evaluating prolidase activity in subjects with non-diabetic MetS may be important as an independent predictor of the disease. However, further studies in larger patient groups are needed to explain the role of serum prolidase activity in the pathogenesis of metabolic syndrome.

\section{Abbreviations}

MetS: Metabolic syndrome; MMPs: Matrix metalloproteinases; OGTT: Oral glucose tolerance test; NCEP: National Cholesterol Education Program; BMI: Body mass index; SBP: Systolic blood pressures; DBP: Diastolic blood pressures; FPG: Fasting plasma glucose; TG: Triglyceride; TAS: Total antioxidant status; TOS: Total oxidative status; OSI: Oxidative stress index; HDL-C: High density lipoprotein- cholesterol; LDL-C: Low density lipoprotein-cholesterol; IFG: Impaired fasting glucose; IGT: Impaired glucose tolerance; TIMP: Tissue inhibitors of metalloproteinase.
}

\section{Competing interests}

The authors declare that they have no competing interests.

\begin{abstract}
Authors' contributions
ST conceptualized the idea for the study, collected the data, performed a literature review, and wrote the manuscript. ST, EO, MAE and NAparticipated in the design of the study, participated in the discussion, and was involved in drafting the manuscript. HK and ST were involved in performing the statistical analysis, participated in the discussion, and were involved in drafting the manuscript. ES and TS participated in the discussion. All the authors have read and approved the final manuscript.
\end{abstract}

\section{Funding}

This research did not receive any specific grant from any funding agency in the public, commercial or not-for-profit sector.

\section{Author details}

${ }^{1}$ Faculty of Medicine, Department of Internal Medicine, Division of Endocrinology, Gaziantep University, 27100, Sahinbey, Gaziantep, Turkey.
${ }^{2}$ Faculty of Medicine, Department of Medical Pharmacology, Harran University, 63300 Sanliurfa, Turkey. ${ }^{3}$ Faculty of Medicine, Department of Internal Medicine, Division of Endocrinology, Harran University, 63300 Sanliurfa, Turkey. ${ }^{4}$ Faculty of Medicine, Department of Internal Medicine, Gaziantep University, 27100, Sahinbey, Gaziantep, Turkey. ${ }^{5}$ Faculty of Medicine, Department of Clinical Biochemistry, Harran University, 63300 Sanliurfa, Turkey.

Received: 17 October 2014 Accepted: 11 December 2014 Published: 17 December 2014

\section{References}

1. Third report of the National Cholesterol Education Program (NCEP), Expert panel on the detection, evaluation, and treatment of high blood cholesterol in adults (Adult Treatment Panel III). Final report. II Rationale for intervention. Circulation 2002, 106:3143-3421.

2. Msra A, Khurana L: Obesity and metabolic syndrome in developing countries. J Clin Endocrinol Metab 2008, 93:9-30.

3. Hopps E, Caimi G: Matrix metalloproteinases in metabolic syndrome. Eur J Intern Med 2012, 23:99-104.

4. Surazynski A, Miltyk W, Palka J, Phang JM: Prolidase-dependent regulation of collagen biosynthesis. Amino Acids 2008, 35:731-738.

5. Zanaboni G, Dyne KM, Rossi A, Monafo V, Cetta G: Prolidase deficiency: biochemical study of erythrocyte and skin fibroblast prolidase activity in Italian patients. Haematologica 1994, 79:13-18.

6. Liu G, Nakayama K, Awata S, Tang S, Kitaoka N, Manabe M, et al: Prolidase isoenzymes in the rat: their organ distribution, developmental change and specific inhibitors. Pediatr Res 2007, 62:54-59.

7. Myara I, Myara A, Mangeot M, Fabre M, Charpentier C, Lemonnier A: Plasma prolidase activity: a possible index of collagen catabolism in chronic liver disease. Clin Chem 1984, 30:211-215.

8. Erbagci AB, Araz M, Erbagci A, Tarakcioglu M, Namiduru ES: Serum prolidase activity as a marker of osteoporosis in type 2 diabetes mellitus. Clin Biochem 2002, 35:263-268.

9. Altindag O, Erel O, Aksoy N, Selek S, Celik H, Karaoglanoglu M: Increased oxidative stress and its relation with collagen metabolism in knee osteoarthritis. Rheumatol Int 2007, 27:339-344.

10. Gejyo F, Kishore BK, Arakawa M: Prolidase and prolinase activities in the erythrocytes of patients with chronic uremia. Nephron 1983, 35:58-61.

11. Demirbag R, Yildiz A, Gur M, Yilmaz R, Elci K, Aksoy N: Serum prolidase activity in patients with hypertension and its relation with left ventricular hypertrophy. Clin Biochem 2007, 40:1020-1025.

12. Erel O: A novel automated method to measure total antioxidant response against potent free radical reactions. Clin Biochem 2004, 37:112-119.

13. Erel O: A new automated colorimetric method for measuring total oxidant status. Clin Biochem 2005, 38:1103-1111.

14. Ozcan O, Gultepe M, Ipcioglu OM, Bolat B, Kayadibi H: Optimization of the photometric enzyme activity assay for evaluating real activity of prolidase. Turk J Biochem 2007, 32:12-16.

15. Gultepe M, Ozcan O, Bolat B, Kayadibi H, Ipcıoglu OM: Measured prolidase activity versus physiological activity of the enzyme: inhibitory effect of proline. FEBS J 2006, 273:75.

16. Myara I, Charpentier C, Lemonnier A: Optimal conditions for prolidase assay by proline colorimetric determination: application to imminodipeptiduria. Clin Chim Acta 1982, 125:193-205.

17. Chinard FP: Photometric estimation of proline and ornithine. J Biol Chem 1952, 199:91-95.

18. Hilali N, Vural M, Camuzcuoglu H, Camuzcuoglu A, Aksoy N: Increased prolidase activity and oxidative stress in PCOS. Clin Endocrinol (Oxf) 2013, 79:105-110

19. Uzar E, Tamam Y, Evliyaoglu O, Tuzcu A, Beyaz C, Acar A, et al: Serum prolidase activity and oxidative status in patients with diabetic neuropathy. Neurol Sci 2012, 33:875-880.

20. Yildiz A, Demirbag R, Yilmaz R, Gur M, Altiparmak IH, Akyol S, et al: The association of serum prolidase activity with the presence and severity of coronary artery disease. Coron Artery Dis 2008, 19:319-325.

21. Camuzcuoglu H, Arioz DT, Toy H, Kurt S, Celik H, Aksoy N: Assessment of preoperative serum prolidase activity in epithelial ovarian cancer. Eur J Obstet Gynecol Reprod Biol 2009, 147:97-100. 
22. Gonçalves FM, Jacob-Ferreira ALB, Gomes VA, Casella-Filho A, Chagas AC, Marcaccini AM, Gerlach RF, Tanus-Santos JE: Increased circulating levels of matrix metalloproteinase (MMP)-8, MMP-9, and pro-inflammatory markers in patients with metabolic syndrome. Clin Chim Acta 2009, 403:173-177.

23. Aquilante $\mathrm{CL}$, Beitelshees $\mathrm{AL}$, Zineh I: Correlates of serum matrix metalloproteinase-8 (MMP-8) concentrations in nondiabetic subjects without cardiovascular disease. Clin Chim Acta 2007, 379:48-52.

24. Miksztowicz V, Muzzio ML, Royer M, Prada M, Wikinski R, Schreier L, Berg G: Increased plasma activity of metalloproteinase 2 in women with metabolic syndrome. Metabolism 2008, 57:1493-1496.

25. Scroyen I, Cosemans L, Lijnen HR: Effect of tissue inhibitor of matrix metalloproteinases-1 on in vitro and in vivo adipocyte differentiation. Thromb Res 2009, 124:578-583.

26. Gummesson A, Hagg D, Olson FJ, Hulthe J, Carlsson LM, Fagerberg B: Adipose tissue is not an important source for matrix metalloproteinase-9 in the circulation. Scand J Clin Lab Invest 2009, 69:636-642.

27. Demeulemeester D, Collen D, Lijnen HR: Effect of matrix metalloproteinase inhibition on adipose tissue development. Biochem Biophys Res Commun 2005, 329:105-110.

28. Belo VA, Souza-Costa DC, Lana CM, Caputo FL, Marcaccini AM, Gerlach RF, Bastos MG, Tanus-Santos JE: Assessment of matrix metalloproteinase (MMP)-2, MMP-8, MMP-9, and their inhibitors, the tissue inhibitors of metalloproteinase (TIMP)-1 and TIMP-2 in obese children and adolescents. Clin Biochem 2009, 42:984-990.

29. Głowińska-Olszewska B, Urban M: Elevated matrix metalloproteinase 9 and tissue inhibitor of metalloproteinase 1 in obese children and adolescents. Metabolism 2007, 56:799-805.

30. Papazafiropoulou A, Perrea D, Moyssakis I, Kokkinos A, Katsilambros N, Tentolouris N: Plasma levels of MMP-2, MMP-9 and TIMP-1 are not associated with arterial stiffness in subjects with type 2 diabetes mellitus. $J$ Diabetes Complications 2010, 24:20-27.

31. Death AK, Fisher EJ, McGrath KC, Yue DK: High glucose alters matrix metalloproteinase expression in two key vascular cells: potential impact on atherosclerosis in diabetes. Atherosclerosis 2003, 168:263-269.

32. Ho FM, Liu SH, Lin WW, Liau CSJ: Opposite effects of high glucose on MMP-2 and TIMP-2 in human endothelial cells. Cell Biochem 2007, 101:442-450.

33. Derosa G, D'Angelo A, Scalise F, Avanzini MA, Tinelli C, Peros E, Fogari E, Cicero AF: Comparison between metalloproteinases- 2 and -9 in healthy subjects, diabetics, and subjects with acute coronary syndrome. Heart Vessels 2007, 22:361-370

34. Derosa G, D'Angelo A, Ciccarelli L, Piccinni MN, Pricolo F, Salvadeo S, Montagna L, Gravina A, Ferrari I, Galli S, Paniga S, Tinelli C, Cicero AF: Matrix metalloproteinase-2, -9 , and tissue inhibitor of metalloproteinase-1 in patients with hypertension. Endothelium 2006, 13:227-231.

35. Fontana V, Silva PS, Belo VA, Antonio RC, Ceron CS, Biagi C, Gerlach RF, Tanus-Santos JE: Consistent alterations of circulating matrix metalloproteinases levels in untreated hypertensives and in spontaneously hypertensive rats: a relevant pharmacological target. Basic Clin Pharmacol Toxicol 2011, 109:130-137.

36. Franz $M$, Berndt $A$, Altendorf-Hofmann A, Fiedler N, Richter $P$, Schumm J, Fritzenwanger M, Figulla HR, Brehm BR: Serum levels of large tenascin-C variants, matrix metalloproteinase- 9 , and tissueinhibitors of matrix metalloproteinases in concentric versus eccentric left ventricular hypertrophy. Eur J Heart Fail 2009, 11:1057-1162.

37. Derosa G, Maffioli P, D'Angelo A, Salvadeo SA, Ferrari I, Fogari E, Gravina A, Mereu R, Palumbo I, Randazzo S, Cicero AF: Evaluation of metalloproteinase 2 and 9 levels and their inhibitors in combined dyslipidemia. Clin Invest Med 2009, 32:124-132.
38. Beaudeux JL, Giral P, Bruckert E, Bernard M, Foglietti MJ, Chapman MJ: Serum matrix metalloproteinase- 3 and tissue inhibitor of metalloproteinases- 1 as potential markers of carotid atherosclerosis in infraclinical hyperlipidemia. Atherosclerosis 2003, 169:139-146.

39. Tabur S, Torun AN, Sabuncu T, Turan MN, Celik H, Ocak AR: Non-diabetic metabolic syndrome and obesity do not affect serum paraoxonase and arylesterase activities but do affect oxidative stress and inflammation oxidative stress and inflammation. Eur J Endocrinol 2010, 162:535-554.

doi:10.1186/1758-5996-6-142

Cite this article as: Tabur et al:: Serum prolidase activity is associated with non-diabetic metabolic syndrome. Diabetology \& Metabolic Syndrome 2014 6:142

\section{Submit your next manuscript to BioMed Central and take full advantage of:}

- Convenient online submission

- Thorough peer review

- No space constraints or color figure charges

- Immediate publication on acceptance

- Inclusion in PubMed, CAS, Scopus and Google Scholar

- Research which is freely available for redistribution

Submit your manuscript at www.biomedcentral.com/submit
C Biomed Central 